\title{
7th Biennial Congress of the Mediterranean Society of Coloproctology (MSCP), Thessaloniki, Greece, April 22-24, 2010
}

\author{
E. Yiannakopoulou
}

Published online: 27 July 2010

(C) Springer-Verlag 2010

I attended a well-organized meeting, with the participation of many speakers from Greece, from the Mediterranean area and from several European countries, despite the ashes of the Icelandic volcano. Topics included the most relevant aspects of coloproctological research. A friendly but frank atmosphere has characterized the comprehensive discussion in every session.

The optimal diagnostic work-up of rectal cancer was stressed. Endorectal ultrasound can accurately predict $\mathrm{T}$ stage in more than $90 \%$ of cases, but it is poor at identifying the $\mathrm{N}$ stage. CT is useful for locally advanced tumors in identifying adjacent organ involvement, but it cannot accurately evaluate the T stages. MRI is able to accurately predict $\mathrm{T}$ stage in 50-90\% of patients but is the most reliable tool to detect the circumferential margin involvement. Accuracy in identifying peritoneal tumor perforation and venous invasion is up to 80 and $83 \%$, respectively (M.G. Pramateftakis, I. Kanellos).

Total mesorectal excision is the gold standard in the treatment of rectal cancer. Additionally, high ligation of colonic arteries and complete mesocolon excision was presented as the optimal surgical technique of right hemicolectomy for colon cancer (M.G. Pramateftakis). Minimally invasive local excision techniques can be applied for early $\mathrm{T} 1$ and $\mathrm{T} 2$ rectal tumors smaller than $4 \mathrm{~cm}$, involving less than $40 \%$ of the lumen circumference, in patients with significant comorbidities to undergo a radical procedure, in symptomatic patients with multi-organ distant metastatic disease and in well-informed patients denying a radical procedure or a stoma (K. Tepetes).

E. Yiannakopoulou $(\bowtie)$

Faculty of Health and Caring Professions, Technological

Educational Institute of Athens, Athens, Greece

e-mail: nyiannak@teiath.gr
Leakage of colonic anastomosis is the most serious complication after colon resection. Early diagnosis and treatment is imperative; minor leaks can be treated conservatively with parenteral nutrition and antibiotics, while operative treatment is needed for major leaks defined by symptoms of peritonitis and septicemia (D. Kanellos).

Loco-regional recurrences of colon cancer (tumor bed, regional nodes, adjoining structures or organs, anastomosis, peritoneum, retroperitoneum, pelvis and surgical scars) after curative colon cancer range between 11 and $40 \%$ and are responsible for $30 \%$ of deaths. The ideal treatment is the complete resection of the recurrence tumor to achieve R0 operation that provides significant long-term survival (A.N. Machairas).

GISTs are the most common mesenchymal gastrointestinal tumors. The most common sites are the stomach and the small bowel. Colorectal GISTs represent 5-10\% of the cases, with rectum being the most common site. Segmental colectomy is the mainstay of treatment for resectable nonmetastatic primary colorectal GIST. Since GIST do not metastasize through lymphatics, lymphadenectomy and mesorectal excision are not necessary. Unresectable tumors could be downstaged with neoadjuvant treatment and restaged for respectability (A. Amato).

First- and second-degree hemorrhoids should be treated conservatively. Stapled hemorrhoidopexy is indicated for third-degree hemorrhoids. The technique is questionable for fourth-degree hemorrhoids, showing a higher long-term recurrence rates than excisional surgery (I. Kanellos, X. Delgadillo).

Obstructed defecation affects nearly $50 \%$ of constipated patients. Symptoms include incomplete and frequent evacuations, straining at stool, self-digitation, perineal and pelvic heaviness, long time spent in toilette. Rectocele and rectal mucosal prolapse are the most common findings, 
although it is not certain if they are the cause or the consequence of obstructed defecation. Occult functional and organic diseases can be underestimated as suggested by the "iceberg syndrome", leading to incorrect therapies. Only $5 \%$ of constipated patients may benefit from surgery (M. Pescatori).

Diverticular disease is the most common organic disorder of the colon and its incidence increases with age. Asymptomatic patients need no treatment. In symptomatic patients with uncomplicated diverticular disease, high-fiber diet is recommended, while the role of antibiotics is still controversial. Acute diverticulitis with mild symptoms and no systemic signs i.e. high fever, tachycardia, malaise and leucocytosis can be treated in an outpatient basis with liquid diet and antibiotics (i.e. ciprofloxacin and metronidazole). Patients with signs of local peritonitis or immunosuppression need urgent hospital admission. Surgical treatment is needed for complications including uncontrolled hemorrhage, obstructive ileus, perforation and sepsis. Controversy still exists concerning the place of prophylactic segmental colectomy and its timing (T. Pavlidis, F. Crescenti).

Medical treatment should be the first-line treatment in all patients affected by chronic anal fissure. Lateral internal sphincterotomy is needed in $15-20 \%$ of patients. Preoperative manometry is selectively performed in any cases at risk for fecal incontinence. After failure of conservative treatment, patients with low anal resting pressure should be treated with $\mathrm{V}-\mathrm{Y}$ or advancement flap anoplasty (G. Pechlivanides).

Restorative proctocolectomy with ileo-anal pouch anastomosis is the treatment of choice for patient with familial adenomatous polyposis and ulcerative colitis (C. Spyridis). Pouchitis is the most common long-term complication, and it is diagnosed when histological acute inflammation is associated with symptoms of urgency, frequency and liquid stools in the presence of endoscopic evidence of inflammation. Cuffitis due to inflammation of retained rectal mucosa may mimic pouchitis (V.N. Papadopoulos).

Recto-vaginal fistulas are not frequent. The etiology includes obstetric trauma, infectious diseases in Bartolini

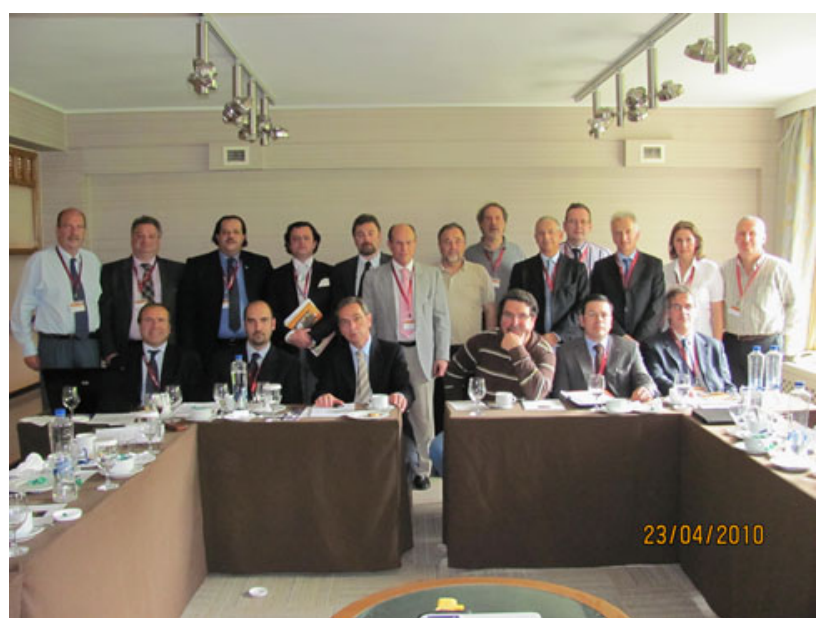

Fig. 1 Governing Council

or anal glands, inflammatory bowel disease, iatrogenic and sexual trauma. Different surgical options have been suggested-fistulotomy and drainage, excision and repair, excision and muscle interposition, flaps, sphincteric reconstruction, colostomy, fibrin glue, fibrin plug-but the optimal treatment is still debatable (X. Delgadillo).

Perianal fistulas can sometimes exhibit no internal opening, no external opening or complex tracts. Superficial, low transphincteric and intersphincteric fistulas are treated by lay-open technique. Loose seton allows time before decision for further management is made. Tight cutting seton is the management of choice for high transsphincteric fistulas. Alternative techniques for high transphincteric fistulas include mucosal advancement flap closure, fistulectomy, fistula excision and sphincteroplasty, injection of fibrin glue and plugs (A. Michalopoulos).

During the congress, the general assembly of the members of the MSCP have elected the next two-years Governing Council of the society (Fig. 1). Our best wishes to the new President I. Kanellos and the councilors for an active and fruitful biennium. The next MSCP meeting will be held in Madrid in 2012, under the organization of M. Devesa. 